\title{
To the special Issue on "Metaheuristics for optimization of complex process engineering"
}

\author{
Jinliang Ding ${ }^{1} \cdot$ Yaochu Jin ${ }^{2}$
}

Published online: 18 July 2016

(C) Springer Science+Business Media Dordrecht 2016

Most modern large-scale complex industrial processes are characterized by multiple conflicting objectives and large amount of uncertainties. Sometimes such processes contain multi-level or multi-stage sub-processes, in which both discrete and continuous decision variables are present. In addition, quality evaluations of such systems may involve computationally very intensive calculations such as finite element analysis. Due to the above challenges, many mathematical programming techniques may no longer applicable. Metaheuristics have shown to be powerful in solving a wide range of global optimization problems. Unfortunately, most recently developed metaheuristics have been tested only on benchmark problems.

This special issue is devoted to the application of metaheuristics to solving real-world optimization problems found in complex process industries. Based on a peer review procedure, ten papers were selected out of fifteen submissions, representing recent advances in applying various metaheuristic optimization methods to complex industrial processes.

The first paper, by Wang et al., is titled "A proactive scheduling approach to steel rolling process with stochastic machine breakdown". The authors address a proactive scheduling problem with stochastic machine breakdown, controllable processing time and deterioration effect considerations commonly seen in steel production. A surrogate-assisted multi-objective evolutionary algorithm based

Yaochu Jin

yaochu.jin@surrey.ac.uk

1 State Key Lab of Synthetical Automation for Process Industries, Northeastern University, Shenyang, China

2 Department of Computer Science, University of Surrey, Guildford, UK on Elitist Non-dominated Sorting Genetic Algorithm is proposed to solve the proactive scheduling problem under consideration. Moreover, a probabilistic sequencing strategy which takes advantage of each job's ability to absorb disruption at low cost is introduced to accelerate the evolutionary search.

The second paper, by $\mathrm{Qu}$ et al., is titled "Solving dynamic economic emission dispatch problem considering wind power by multi-objective differential evolution with ensemble of selection method". The authors propose a modified version of multi-objective differential evolution to tackle the extended dynamic economic emission dispatch problem, in which fuel costs and pollution emission are the two objectives to be minimized. The problem is a time-varying, nonlinear and highly constrained multi-objective optimization problem. An ensemble of selection method is adopted in the algorithm to enhance the performance. To deal with the constraints, both real-time output adjustment and penalty factor methods are considered.

The third paper, by Kordestani et al., is titled "New measures for comparing optimization algorithms in dynamic environments". The authors present a set of new measures for comparing optimization algorithms in dynamic environments. A comparative study is conducted using a number of state-of-the-art algorithms for dynamic optimization on the Moving Peaks Benchmark, along with several other performance measures, to demonstrate the relative advantages of the introduced measures. It is hoped that the proposed new measures are able to provide a more complete comparison of dynamic optimization algorithms.

The fourth paper, by Qiao et al., is titled "A Regularization-reinforced DBN for Digital Recognition". The paper reports an optimization approach to obtaining the minimum-magnitude weights of regularization-reinforced deep belief network to enhance its performance. A special 
regularization-reinforced term is developed to reduce the weights in the unsupervised training process. The network is successfully applied to hand-written number classification and water quality prediction.

The fifth paper, by Ye et al., is titled "A hybrid instanceintensive workflow scheduling method in private cloud environment". The authors aim to solve the problem of instance-intensive workflow scheduling in private cloud environment. A scheduling optimization model considering the communication time between tasks is formulated, with its objective being to minimize the execution time of all workflow instances. Then, a hybrid scheduling method based on the batch strategy and an improved genetic algorithm termed fragmentation based genetic algorithm (FBGA) is proposed according to the characters of instance-intensive cloud workflow, where task priority dispatching rules are also taken into account.

The sixth paper, by Guo et al., is titled "A dynamic SVR-ARMA model with improved fruit fly algorithm for the nonlinear fiber stretching process". In this paper, the authors develop a support vector regression model using industrial data for modelling the nonlinear fiber stretching process in fiber production. The parameters of the model are optimized using an improved fruit fly algorithm. Taking into account the high cost and accurate precision of the fiber stretching process, a time series autoregressive moving average model is introduced to reduce the prediction error of the support vector regression model.

The seventh paper, by Fu et al., is titled "A decomposition based multi-objective genetic algorithm with adaptive multi-population strategy for flowshop scheduling problem". The authors introduce a new decomposition based multi-objective genetic algorithm with adaptive multi-population strategy to address multi-objective permutation flowshop scheduling problems. A subpopulation construction method is employed to construct multiple subpopulations adaptively to optimize their corresponding subproblems in parallel.

The eighth paper, by Wang et al., is titled by "Hyperpath-based vehicle routing and scheduling method in timevarying networks for airport shuttle service". This paper suggests a vehicle routing and scheduling method, where the time-varying feature enables the traveller to select a direction among all the Pareto-optimal paths at each node in response to the knowledge of the time window demands. To obtain the Pareto-optimal paths (called hyperpaths), an exact algorithm is designed for addressing the bi-criteria shortest path problem. A computationally efficient bound strategy is reported on the basis of passenger locations, pickup time windows and arrival time windows. The vehicle routing and scheduling problem is solved by a proposed heuristic algorithm combined with a dynamic programming method.

The ninth paper, by Sang et al., is titled by "Selfadaptive fruit fly optimizer for global optimization". The authors describe a self-adaptive fruit fly optimization algorithm for solving high-dimensional global optimization problems. The proposed approach tries to modify the control parameters during the optimization by taking the actual search process into account to guide its search along an appropriate decision variable from its previous experience in generating promising solutions. It has been shown to be able to significantly improve the search capability of the fruit fly optimization.

The tenth paper, by Wang et al., is titled "On an integrated approach to resilient transportation systems under emergency situations". An integrated approach to the evacuation problem under an emergency situation for transportation systems is presented in this paper. The approach is based on a view that a service system has two subsystems: infrastructure and substance, where the overall objective function in the problem model is a minimum transportation time. The approach attempts to integrate infrastructure design and substance flow planning to improve the evacuation performance. A discrete variable Particle Swarm Optimization combined with a classical algorithm is applied to solve the problem model. Since recovery of a damaged transportation (damaged road in this case) is implied, the proposed approach has some significant implication to resilience engineering of a service system as well.

We would like to thank Prof Joost Kok, the Editor-inChief, for giving us the opportunity to guest-edit this special issue and great assistance during the process. We are equally grateful to all authors who submitted their papers to the special issue and to reviewers for their timely and insightful review that have greatly helped improve the quality of the papers presented here. 